\title{
FMH-Strukturreform vor der zweiten Lesung in der Ärztekammer
}

Strukturen allein machen noch keine Politik. Gute Strukturen und Abläufe können aber wesentlich mithelfen, wirkungsvolle Berufspolitik zu machen. Die Ärztekammer hat im Juni 2004 ein Leitungsgremium für die FMH-Strukturreform* gewählt. Die zweite, definitive Lesung der neuen Statuten und eine Revision der WBO wird nun an der Ärztekammer vom 18. und 19. Mai 2006 stattfinden. Das Wichtigste in Kürze:

Eine 32köpfige Delegiertenversammlung (DV) ersetzt die bisherige Präsidentenkonferenz (PK). Sie soll die Arbeit des Zentralvorstandes begleiten und legitimieren. Die DV wird rund sechsmal im Jahr tagen, also deutlich häufiger, als es die PK tat. Die Delegierten werden von den Dachverbänden nominiert, und ihre Wahl wird durch die Ärztekammer bestätigt. Das Prinzip «Ablösung der PK durch die Delegiertenversammlung» erscheint heute unbestritten. $\mathrm{Zu}$ diskutieren geben wird naturgemäss, welches ein anerkannter Dachverband ist («fachliche oder regionale Zusammenschlüsse von Ärzteorganisationen»), und welcher wieviele Delegierte nominieren kann. Die Delegierten sollen dann aber in der DV nicht ihre Klientel, sondern das Gemeinwohl der FMH vertreten!

Die Kommission für Weiter- und Fortbildung (KWFB) soll künftig die Weiterbildungsprogramme auf Vorschlag der Fachgesellschaften selbst beschliessen. Heute tut dies noch der ZV. Die KWFB soll zudem kleiner werden. Vorgeschlagen sind je ein Delegierter pro Fachgesellschaft mit gewichtetem Stimmrecht je nach Grösse der Fachgesellschaft (statt bisher 2 bis 10 Delegierte); die Fakultäten, der VSAO und der VLSS sollen je 2 weitere Delegierte entsenden.

Ausserhalb der Strukturreform im eigentlichen Sinn schlägt das Leitungsgremium vor, die Vereinigung der leitenden Spitalärzte der Schweiz VLSS neu als Basisorganisation für die Chef- und leitenden Ärztinnen und Ärzte einzusetzen. Allerdings sollen die Chefärztinnen und Chefärzte weiterhin zusätzlich auch Mitglied in der kantonalen Ärztegesellschaft sein, wobei die bestgeeignete Form der kantonalen Mitgliedschaft im Kanton allenfalls neu auszuhandeln ist.
Zudem werden weitere Anträge auf Einsitz mit Stimmrecht in der Ärztekammer erwartet, beispielsweise seitens Ärztinnen Schweiz (MWS), der Schweizerischen Belegärztevereinigung (SBV) sowie der Swiss Federation of Specialities in Medicine (SFSM).

Wie geht es weiter? Wenn die Ärztekammer die Reform in der vorliegenden Fassung gutheisst, sieht der Fahrplan so aus:

- Die KWFB kann schon ab etwa Spätsommer 2006 in neuer Zusammensetzung und Kompetenz tagen (konkret: nach Ablauf der Referendumsfrist für die revidierten Statuten und die WBO).

- Nach der Statutenrevision wird die Geschäftsordnung (GO) der FMH revidiert. Diese Arbeit wird noch im 2006 an die Hand genommen. Auch die GO muss dann von der Ärztekammer verabschiedet werden.

- Bevor die Delegiertenversammlung erstmals tagen (und die PK real ablösen) kann, muss die Ärztekammer die Delegierten bestätigen. Dies kann an der nächsten Ärztekammersitzung nach dem Mai erfolgen. Soll auch noch die GO-Revision abgewartet werden? Die Statutenbestimmungen für die DV sind recht detailliert, so dass - den entsprechenden politischen Wunsch vorausgesetzt - die DV auch schon nach Bestätigung der Delegierten durch die Ärztekammer tagen kann, ohne dass man Angst vor einem Chaos haben müsste.

- Die neuen Bestimmungen sollen nicht in Stein gemeisselt sein. Es ist geplant, nach rund zwei Jahren Erfahrung eine Zwischenbilanz zu ziehen.

Soweit der Überblick. Ich bin gespannt auf die Entscheide der Ärztekammer und darauf, wie sich die Zusammenarbeit zwischen der neuen DV und dem Zentralvorstand gestalten wird.

Hanspeter Kuhn, Fürsprecher, stv. Generalsekretär

\footnotetext{
Interessierte können die der Ärztekammer verschickten Unterlagen zur Strukturreform auf der Website der FMH einsehen: www.fmh.ch $\rightarrow$ Über uns $\rightarrow$ Strukturreform.
} 